\title{
The Optimal Emission Decisions of Sustainable Production with Innovative Baseline Credit Regulations
}

\author{
SungYong Choi ${ }^{1}$, KyungBae Park ${ }^{2, *(\mathbb{D})}$ and Sang-Oh Shim ${ }^{3} \mathbb{C}$ \\ 1 Division of Business Administration, Yonsei University, 1 Yonseidae-gil, Wonju, Gangwon-do 26493, Korea; \\ sungyong.choi@yonsei.ac.kr \\ 2 Department of Business Administration, Sangi University, 83 Sangjidae-gil, Wonju, \\ Gangwon-do 26339, Korea \\ 3 Department of Business Administration and Accounting, Hanbat National University, 125 Dongseo-daero, \\ Yuseong-gu, Daejeon 34158, Korea; soshim@hanbat.ac.kr \\ * Correspondence: kbpark@sangji.ac.kr; Tel.: +82-33-730-0311
}

Received: 28 December 2018; Accepted: 2 March 2019; Published: 18 March 2019

check for updates

\begin{abstract}
In the era of the fourth industrial revolution, the international community is striving to establish a coordinated system to prevent fatal climate change in a global sense. As a result of such changes in business environments, a new issue, sustainability, has recently presented a paradigm shift and new research opportunity in which the theories and practices in traditional production and operations management are being reinterpreted and reapplied in relation to this emerging issue. Under this research background, we consider an optimal emission-trading problem under a cap-and-trade (CAT) emission regulation when the customers' demand is given as an arbitrary probability distribution. Such a CAT approach to reduce the amount of emissions is a normative system for the sustainable production of manufacturing firms, which is also closely related to a well-known open innovation in literature of inventory management. Then, we formulate two stochastic inventory optimization models, which can be applied immediately for two famous CAT policies that exist in reality. In particular, our objective is to draw theoretical and practical implications for baseline credit emission regulations, which are innovative and government-led emission regulation policies, with a well-known newsvendor analysis. For our analytical results, we first show that our objective functions are piecewise linear and (quasi)-concave. Thus, it is found that there exists a unique optimal solution to the problem. Second, we successfully obtain the closed-form optimal solutions for the two models considered. Finally, we conduct a sensitivity analysis through a comparative static analysis to examine how the model parameters can affect the optimal solution in each model. All these analytical results and implications are consistent with previous studies in the literature, as well as with our insights for the models.
\end{abstract}

Keywords: cap-and-trade system; baseline credit regulations; newsvendor problem; emission right trading; inventory management; sustainable production; open innovation

\section{Introduction}

As environmental concerns grow globally, it is emphasized that appropriate international controls are required to prevent irreversible and lethal climate change and preserve the global ecosystem [1]. In the era of the fourth industrial revolution, the international community has been working together to establish an international cooperation to continuously reduce carbon emissions [2]. Therefore, there is a closely tied relationship between eco-friendly production and sustainability in manufacturing and 
service firms $[3,4]$. Although such a close relationship between them has become more prominent in recent years, it is not a completely new phenomenon.

In recent years, researchers and practitioners in the field of production and operations management (POM) have been studying increasingly challenging situations about how to combine their traditional efficient and optimal production issues with a new emerging issue of sustainability. In other words, as the order of the global economy becomes more complicated, it is evident that only an immediate profitability does not guarantee the perpetual growth of companies and the economy. As a result, people began to reflect deeply on the future of humans and the earth. Because of such changes in business environments, a new issue, sustainability, has recently presented a paradigm shift and new research opportunity in which the theories and practices in traditional production and operations management are being reinterpreted and reapplied in relation to this emerging issue. The authors of [4] have presented three specific research agenda that have been actively studied for sustainable production in the field of POM over the last 20 years, which are green-product and process development, lean and green POM, remanufacturing, and closed-loop supply chains. In contrast, the optimal emission decision modeling under the emission trading system, which is the subject of this study, is a relatively new research subject in the field of POM. Therefore, this subject needs to be studied more actively in the near future.

Dating back in the past, more than 160 countries reached the United Nations Framework Convention on Climate Change (UNFCCC) agreement in 1992, the first international treaty to control carbon emissions [5]. However, the role of the UNFCCC is quite limited in that it is just a preliminary and basic agreement for transnational global cooperation against climate change. After then, the specific details of the UNFCCC reached a significant consensus in the Kyoto Protocol in 1997 [6]. More specifically, in the Kyoto Protocol, participating major developed countries are classified as Annex 1 groups. Then, among these countries, there have been agreements to impose legally binding emission targets for various pollutants, including carbon dioxides. Although the United States and Australia failed to ratify the Kyoto agreements among the major industrialized nations, the agreements became effective in 2005. The Kyoto agreements first proposed a number of trading mechanisms, including a flexible mechanism, as an international emissions trading system. Thus, the Annex 1 Group countries allowed trading on their assigned emissions credits using the international Emissions Trading (ET), ensuring compliance with pre-established emission limits in line with market mechanisms.

These Kyoto agreements can be considered to have implemented a cap-and-trade (CAT) system in that they allocate country-specific emission limits to Annex 1 countries. In the CAT system, governments set an overall limit on the emission amounts of individual polluters by reference to past emissions records, and polluters are allowed to emit within that limit $[7,8]$. As these emission rights can be traded freely, each polluter can choose his optimal emissions decision based on his own economic incentive-whether to sell the remaining amount of emission within the limit, or to purchase the excess emission amount from other polluters. The optimal strategy of these polluters is influenced by external circumstances such as the price of the extra emission quota, as well as the internal situation such as the emission cost reduction of the polluters. In the literature, the CAT system has been known to lead to an efficient way of production in manufacturing firms as well as a socially sustainable choice of production from an economic point of view. Owing to these reasons, the CAT system can be compared to a command-and-control system, one of the other normative emissions limit mechanisms [9].

This command-and-control system is one of the traditional approaches to pollution reduction and is a way to specify and enforce emission limits for each polluter [10]. Command-and-control systems do not allow the transfer of pollutant reduction responsibilities to companies that can do this more efficiently and at a lower cost [11]. In other words, the command-and-control system is a way to enforce strict regulations based on quantity, and the resulting system cost increases. On the other hand, the CAT method is based on price and focuses on economic incentives [12]. In addition, from a modeling standpoint, command-and-control can be applied only if the end customer's demand is 
known as a constant, because it aims to control emissions completely. In contrast, the CAT system can be applied to cases where the demand of end customer is both deterministic or probabilistic. For these reasons, the CAT system has been confirmed to be useful both academically and practically, and has been actively studied.

In this way, for the CAT and command-and-control systems, two normative systems for reducing emissions, it can be argued that these two systems have the following logical links with open and closed innovation. First, open innovation aims to open up the innovation process and utilize internal and external resources in a balanced way to improve the operational activities of the companies. By doing so, we intend to maximize the creation of value added by reducing the cost of innovation and increasing the likelihood of success. In other words, the open innovation that Chesbrough originally claimed is to accelerate internal innovation and expand the external utilization to the market of innovation by appropriately utilizing the intellectual flow of knowledge and the outward knowledge flow [13]. The process of open innovation is about how companies combine internal and external ideas to build architectures and systems through their business models. In contrast, the concept of closed innovation emphasizes that a successful innovation process requires internal control of key business activities, such as production, as well as ideas for innovation. In that sense, when an innovation process is applied for an emissions trading scheme, the CAT system can then lead to total optimality in the emissions decision by purchasing extra or insufficient emissions through trading in the market. This means that the CAT system can actively utilize external resources by purchasing or providing emissions credits to external vendors. Thus, the CAT system is equivalent to applying the innovation process to an emissions trading scheme from an open innovation approach. On the other hand, the command-and-control system corresponds to the application of the closed innovation approach in an emissions trading scheme, in that companies make their own optimal emissions decision in an internal basis.

In the literature, there exist two innovative regulation approaches, pollution tax regulations (PTR) and baseline credit regulation (BCR), as the practically feasible regulation policies for implementation of the CAT system $[10,12,14]$. Indeed, each of the two approaches functions as a tax (or subsidies for negative values) that is affected by the costs and reduction amounts of pollution abatement. Then, operational optimization models can be considered for individual companies based on each regulatory approach as follows. First, the purpose of PTR is to reduce the pollutant emissions by imposing per unit cost on the pollutants generated from firms' production activities. When the pollutant emissions exceed or fall below the emission limit, the difference can be traded in the market. Second, under BCR, only if the pollutant emissions exceed the predetermined baseline, the difference can be purchased from other polluters. That is, if the emission amount is less than the baseline, the difference will be discarded. Note that which of these two approaches policymakers choose will be affected by the nature of the pollutant [15]. For example, in the case of carbon dioxide, there is very little difference in the environment depending on the place where it is discharged. Therefore, the emission reduction cost per unit amount shows little difference according to the place of emission, so the PTR is more appropriate because the place of emission is not a big issue from the environmental point of view and the market price of the emission trading can be determined relatively uniformly. On the other hand, the environmental impacts of sulfur dioxide, mercury, and nitrogen compounds are very different from region to region. Thus, the price of emission trading may not be the same in each region, and then $\mathrm{BCR}$ may be a better approach in a CAT system [15,16]. In addition, the authors of [15] pointed out policy choices and economic factors as the reasons for these price differences. Among these, policy choices are actually determined by governments, which is related to how the CAT system is structured and which specific factors are included in the design of the system. Economic factors include development of environmentally friendly technologies and market penetration, or government funding policies and tax incentives. Meanwhile, the authors of [16] illustrate examples in which some pollutants (e.g., dioxins, obsolete pesticides) have more severe environmental problems in the world. 
In this study, we focus on the BCR approach in the CAT system to provide a stochastic optimization inventory model, in contrast to the previous studies in the literature that focus on the PTR approach. Thus, our goal is to provide an optimization model with the BCR approach that maximizes the operational efficiency of individual companies and to derive the theoretical implications and insights. The main research questions of this study are as follows: (1) how can BCR methods in a CAT system be formulated into an inventory management optimization model? (2) does there exists a unique optimal solution in the BCR optimization models? (3) how does the optimal solution change when the values of the model parameters vary? To this end, we provide the optimization model and analyze it with a sensitivity analysis.

The remainder of the study is composed as follows. In Section 2, we discuss the theoretical background and examine CAT carbon emission approaches and their modeling results that have been studied in the literature. In Section 3, we derive two optimization models with the BCR approach and analyze the models. We obtain a closed-form optimal solution for each BCR model and conduct a sensitivity analysis for the optimal solution. For this purpose, we provide our comparative static analysis to examine the impacts of model parameters on the optimal solution. Finally, in Section 4, we summarize our main results and contributions, and presents some limitations and future directions of research.

\section{Literature Review}

In response to these growing international interests, the issues of energy efficiency and environmentally friendly production have been actively studied in the literature from engineering and economics perspectives. Such examples of engineering issues include energy-inefficient equipment and facility replacement, product and packaging redesign, use of renewable energy, and energy-saving process processes [17]. The authors of [18] develop an innovative initial allocation scheme of tradable carbon emission allowance for manufacturing companies in Shenzhen, China. The basic idea of the scheme is to determine allowance allocation by actual output and carbon intensity. Then, they establish a two-step allocation procedure and introduce a competitive game mechanism to allocate allowance amounts. On the other hand, from the economic point of view, designing international trade mechanisms for emissions trading has been frequently studied in the literature. Then, the focus was given to the policy effects on eco-friendly regulations. In particular, these policy regulations can be categorized into two groups-based on prices and quantities, respectively. Note that price-based regulation mainly addresses imposing taxes on carbon emission amounts, while quantity-based regulation allocates maximum allowable amounts to emissions by which companies can trade the emission rights in the market [17,19-21]. From our broad literature review, it is found that there are a relatively small number of papers to analyze operational efficiency and optimization models with the CAT system. However, in recent years, some papers have been studied, including this work, on optimal modeling of the emission trading decision from a perspective of sustainability. Next, from an analytical point of view, several previous studies of sustainable CAT optimization models can then be divided into two groups-deterministic and stochastic models.

First, in the deterministic models, the authors of [8] set up an inventory management model with the carbon emissions trading regulations. More specifically, they applied the classic economic order quantity (EOQ) model to formalize the carbon emissions problem and, as a result, proposed a modified EOQ model including a carbon balance constraint. They also obtained a closed-form solution for optimal inventory level and performed a comparative static analysis of the optimal solution for the model parameters including carbon emission limits and prices. Finally, they confirmed analytical results through numerical analysis and provided interesting intuitions and insights into their research model. The authors of [17] presented several deterministic optimization models for various regulatory methods related to carbon emissions and formalized them into multi-period mixed integer linear programming (MILP) models. They then conducted extensive numerical analysis and, as a result, presented meaningful implications and insights along with economic explanations. The authors 
of [22] considered a two-echelon supply chain management (SCM) model with one retailer and one manufacturer, respectively. They assumed that both retailer and manufacturer could invest in greening efforts and, in proportion to this, increase the demand from end customers. They then compared the sensitivity of the optimal greening efforts and greening costs in centralized and decentralized supply chains. Finally, among various methods of SCM coordination considered, a two-part tariff contract was found to coordinate the given SCM model. The authors of [23] consider two carbon policies, carbon CAT and carbon tax regulation, under exogenous and endogenous sustainable trade credit, which is one of the most commonly used short-term funding methods. Then, under these model settings, the structural characteristics of the optimal solutions are analyzed with a numerical study. The authors of [24] analyze how a retailer determines her optimal ordering quantity with a CAT system and trade credit. Then, they show that the CAT system and trade credit jointly affect the retailer's optimal ordering quantity. The authors of [25] provide an optimization model and its algorithm for a closed-loop production routing problem with simultaneous pickups and deliveries (PRPSPD) in a CAT system for carbon emission. Specifically, they formulate the CAT with PRPSPD as a mixed-integer linear programming problem with a branch-and-cut guided search algorithm. Finally, they conduct an extensive computational experiment to evaluate the performance of their algorithm and develop managerial implications through their sensitivity analysis. The authors of [26] consider a Stackelberg game of a dual-supply chain, which contains an online direct channel and offline traditional retail channel. Then, it discusses the pricing decision in the supply chain as well as the impact of the CAT system on emission reduction policies. Finally, it is also shown how the impact of customers' low-carbon preference affects the optimal pricing and emission reduction amount. The authors of [27] handle material purchasing in terms of sustainability. Then, they develop a bi-objective EOQ model of transportation and lot-sizing when seeking a Pareto frontier with a CAT mitigation policy. Finally, they conduct a parametric analysis, based on real industrial cases, to show a sensitivity analysis of the results.

Second, in the stochastic model, the authors of [28] provided an excellent summary of the previous works in this research stream. As can be inferred from the above summary, the previous CAT stochastic models, including the work of [29], focused on sustainable SCM coordination rather than determining the optimal production level for individual manufacturing firms. On the other hand, this study has significant theoretical contributions in that it analyzes the optimal production amount of individual manufacturing firms with BCR by applying the well-known newsvendor models in POM literature.

The newsvendor model has initiated since the seminal work of [30], which corresponds to a classic model of the literature at stochastic inventory management. More specifically, it is an optimization model for determining the optimal inventory level in a single-selling season in which the demand for end customers is not fixed but only as a form of a probability distribution. It is effectively modeling the reality of inventory management decision making and can find its applications in various demand-driven manufacturing and service supply chains. In other words, the newsvendor model corresponds to a mathematical optimization model that effectively compresses the real inventory decisions faced by the inventory managers as a trade-off between overage and underage costs. In addition, from an analytical point of view, one of the major advantages of the newsvendor model is that it can be formulated as an unconstrained optimization model. Furthermore, when there are no additional (e.g., budget or resource) constraints reflecting the actual inventory management situation, the newsvendor model has a closed-form optimal solution with arbitrary demand distributions, which provides simple yet powerful insights into the model. While the work of [28] is one of the most similar studies in the literature, it considered and analyzed the impacts of a PTR in a CAT system, which is different from our study.

Since then in this research stream of inventory management literature, various types of expanded and modified newsvendor models have been actively studied in this research stream [31,32]. Here, the authors of [31] pointed out that newsvendor problems have a wide applicability in manufacturing and service companies as product life cycles tend to decrease, which is what happened in recent 
time. Then, the authors of [31] categorized existing newsvendor models into 11 groups, each of which theoretically belong to a certain class of newsvendor problems. For more details, please refer to the work of [31]. Next, the authors of [32] intend to review the previous works in the literature with three research streams, which are customer demand types, supplier pricing policies, and buyer risk profile. First, the analysis of customer demand types is related to how the optimal ordering amount is influenced when customers' demand is a function of (1) market price; (2) marketing effort; and (3) stocking quantity. In (1), unlike the classic newsvendor problem where selling price is given exogenously, price-dependent demand is assumed in the model. Then, the existing studies consider additive and multiplicative demand with a random term. Each of the works studied shows structurally different analytical results depending on each model and demand type. Next, in (2), it is assumed that various marketing activities may affect customers' demand. This stream of research is very important in our study because such impact of marketing efforts can be similarly extended to our subject, newsvendor problems with sustainability, like the impact of greening efforts on demand. Under this setting, the analytical results in the previous studies generally show that an increased mean demand due to marketing efforts steps up optimal ordering quantity. However, the effect of a decrease in demand variability is indeterminate because of marketing efforts. In (3), the stocking amount displayed in retail stores is assumed to influence the ordering decision. Second, the analysis of supplier pricing policies considers a supply chain coordination through supplier pricing. Then, it also studies various types of quantity discount models such as linear quantity discount, all-units quantity discount, and incremental-unit quantity discount models. In the last case, the analysis of buyer risk profile is related with various risk preferences of newsvendors. That is, the most common risk preference in the literature of inventory management is risk neutrality, which is equivalent to the expected-value optimization model in a modeling perspective. However, some inventory managers may not be risk-neutral. Rather, they may prefer risk aversion or risk seeking. After examining the previous studies and empirical evidence, general utility functions, mean-variance, and coherent risk measures have been discussed. Although each model setting has different analytical results, a general finding is about how the degree of risk aversion affects the optimal ordering amount. For this effect, when the unit shortage cost, due to stockout penalty, is less than the given threshold value, risk aversion leads to the lower optimal solution. However, if the unit shortage cost is sufficiently high, risk aversion generally increases the optimal solution.

In the last case, there are also a few more works in the literature to study the regional diversity of the environmental impacts in some pollutants, including sulfur dioxide, mercury, and nitrogen compounds, as follows. The authors of [33] estimated the amount of sulfur dioxide emissions at world and regional levels, caused by anthropogenic sources from 1980 to 2000. As a result, there has been no significant change in anthropogenic annual sulfur dioxide emissions from the world for 20 years, but there has been a major regional change. Remarkably, while the share of North America in sulfur dioxide emissions has been greatly reduced, Asia's share has continued to increase steadily, which seems to be due to Chinese economic growth. This study has a major contribution in providing a clear pattern of data on the impact of human economic activity on the climate. Then, the authors of [34] extended the investigation period for sulfur dioxide emissions and estimated the amount of sulfur dioxide emissions caused by anthropogenic sources from 1850 to 2005. As a result, in addition to China's economic growth, the main reasons for the recent increase in sulfur dioxide emissions are international shipping and global economic growth, mainly in developing countries. In addition, it is pointed out that the volatility of the sulfur dioxide emissions is relatively stable at the world level, while the regional variability is increasing. The authors of [35] examined the environmental impact of mining activities on soil and superficial waters as well as local atmosphere associated to an abandoned $\mathrm{Hg}$ mine in Spain. Then, waste generated and accumulated on the ground and water surfaces, potentially causing acid rains. As a result, even the old metallurgical waste has a significantly harmful effect on their environments. The authors of [36] point out that the emission of nitrogen compounds has a serious adverse effect on climate change as a greenhouse gas (GHG) when their 
concentration exceeds some certain level. In particular, the need for managing the animal waste problem is critical to develop sustainable solutions for this problem. In order to resolve the problem, all of the environmental issues including air, water, and soil should be examined for a long-term, comprehensive strategy. Accordingly, some countries such as Europe and Canada have raised issues related to technically and economically developing feasible solutions for animal waste problems.

\section{Problem Formulation}

In this section, we describe our mathematical model with the BCR approach as follows. Initially, an inventory manager faces purchasing costs $c$ for the raw material and then finally resells her finished products to customers at an increased price $p$. Then, she needs to determine her optimal production quantity $x$ to satisfy customers' demand. Note that we assume our problem is a single-echelon model to simplify manufacturing environments.

With a BCR problem setting, let us denote $\alpha$ and $K$ as the unit trading value for emission right and the initial permissible emission cap, respectively. In addition, the emission amount is initially zero when the production quantity is zero. Once production occurs, the (fixed) base emission amounts of $a$ are generated, and then emissions increase in proportion to production quantity with a constant coefficient $b$ for the extra emission charge per unit item produced. Note that total emission amount $e(x)$ is assumed to be a function of production quantity $x$ as $a+b x$ only except for an instantaneous jump of size $a$ at the level of zero production. Note that in order to avoid a trivial solution, it is reasonable to restrict the range of the optimal production quantity as a set of positive values without loss of generality. Finally, the total emission amount $e(x)$ can be represented simply as $a+b x$, which is a linear function of $x$ for any positive production quantity.

Next, if the total emission amount is larger than the initial permissible emission cap (i.e., $a+b x \geq K)$, the inventory manager should purchase and then fulfill the difference $a+b x-K$ from available emission right suppliers with the unit trading value $\alpha$. Otherwise, if the total emission amount is below the emission cap as $a+b x<K$, the remaining emission right would be discarded.

In the literature of inventory management, many academic works, including newsvendor models, have considered an uncertain demand to incorporate the imbalance between supply and demand. According to this tradition, an inventory manager faces a stochastic demand $D$ for a single product with its cumulative distribution function $F_{D}(\cdot)$ and probability density function $f_{D}(\cdot)$. In this problem, this inventory manager needs to determine his/her optimal production quantity $x$ before the realization of the demand.

Then, the inventory manager has a profit function as follows:

$$
\Pi(x, D)=p * \min \{D, x\}-c x+v(x-D)^{+}-s(D-x)^{+}-\alpha(a+b x-K)^{+},
$$

where $(\cdot)^{+}=\max (0, \cdot)$. In Equation (1), the first term implies the total revenue for the inventory manager. As the initial on-hand inventory is zero, the inventory manager can sell no more than his on-hand inventory even if the on-hand inventory is smaller than the realized demand. In the next, the second term is the purchasing costs for raw material to produce $x$ units. Then, the third and fourth terms are salvage value and stockout penalty costs, respectively. In particular, if the realized demand is higher than the production quantity, this item is short of the difference $D-x$ incurring shortage cost $s$ per unit. These shortage costs per unit when sold out are related to the costs that occur due to stockout penalty. Then, lost sale is a common practice in a manufacturing setting. If excess demand is lost, the stockout cost means the opportunity cost of unrealized profit, mainly due to lack of inventory. Thus, such stockout penalty costs are generally proportional to the amount of the unmet demand [37]. Similarly, if the realized demand is lower than the production quantity, the inventory manager can get only the salvage value $v$ per unit. That is, a unit salvage value implies perishability of the item produced. Such perishabiliy often occurs not only in items that can easily depreciate in value, such as food, but also in manufacturing items, where technical obsolescence can occur. Next, the fifth term 
indicates a trading value for the emission shortfall amounts with the emission cap $K$ to incorporate the BCR system. In the last, we also need to have the conditions, (1) $\alpha \geq 0, b \geq 0,0 \leq a \leq K$ and (2) $0<v<c<c+\alpha b<p$, to avoid trivial solutions with classic newsvendor problems and the BCR approach. Finally, our objective function for the inventory manager is to maximize the expected profit, which is given as follows:

$$
\max _{x \geq 0} \mathbb{E}[\Pi(x, D)]
$$

Currently, Equation (2) implies an unconstrained stochastic optimization model only except for the non-negativity condition $x \geq 0$ in a modeling perspective. In the last, similar to the work of [28], let us classify the model parameters into two groups. The first group covers $p, c, v$, s, which form the first four terms in Equation (1) to represent the basic newsvender problem. In comparison, the second group includes $a, b, K, \alpha$, which are in the fifth term in Equation (1) to specify the BCR system. From now on, let us denote that the first four terms and the last (fifth) term of Equation (2) are newsvendor and BCR terms, respectively. Similarly, if the BCR term is replaced by $-\alpha(a+b x-K)$, Equation (2) is equivalent to the objective function of Model 2 in the work of [28] when $\lambda=1$, which we call a PTR term. From that sense, this work is a direct extension of the work of [28] when the BCR term is replaced by the PTR term with $\lambda=1$. Next, let us call the first and second groups of the model parameters as newsvendor and CAT parameters because both the BCR and PTR terms can be represented by $a, b, K, \alpha$.

\section{Mathematical Analysis}

Now, we begin our analysis with the results of Model 2 in the work of [28] with $\lambda=1$, which we call Model 0 as a base model of the study. For Model 0 , we redefine a new profit function $\Pi_{0}(x, D)$ as follows.

$$
\Pi_{0}(x, D)=p * \min \{D, x\}-c x+v(x-D)^{+}-s(D-x)^{+}-\alpha(a+b x-K) .
$$

That is, the difference between $\Pi(x, D)$ and $\Pi_{0}(x, D)$ is only at the last term in which $-\alpha(a+b x-K)^{+}$ is replaced by $-\alpha(a+b x-K)$. Then, we set up Model 0 by taking an expected-value operator over $\Pi_{0}(x, D)$, which is as follows:

$$
\text { Model } 0: \max _{x \geq 0} \mathbb{E}\left[\Pi_{0}(x, D)\right]=\mathbb{E}\left[p * \min \{D, x\}-c x+v(x-D)^{+}-s(D-x)^{+}-\alpha(a+b x-K)\right] \text {. }
$$

Then, the (risk-neutral) objective function of Model 0 consists of newsvendor and PTR terms, while that of Model 1 is the expected sum of newsvendor and BCR terms. From that sense, Model 0 lays a theoretical background for our model as a preliminary result. In addition, the $B C R$ term in Model 1 shows an asymmetric cost structure at a kinked point $x=(K-a) / b$, while the PTR term in Model 0 represents a symmetric cost structure in $x$. Note that only Models 1 and 2 are our research model, while Model 0 is a reference model of the study because Model 0 is a special case of Model 2 in the work of [28]. Then, we will show how Models 1 and 2 are closely related to Model 0, but different from one another.

Lemma 1. In Model 0 , the objective function $\mathbb{E}\left[\Pi_{0}(x, D)\right]$ is concave for all $x$ and there exists a unique optimal order quantity $\hat{x}_{0}(\alpha)=F_{D}^{-1}\left(\frac{p+s-c-\alpha b}{p+s-v}\right)$. In addition, if $\alpha=0, \hat{x}_{0}(0)=F_{D}^{-1}\left(\frac{p+s-c}{p+s-v}\right)$, which implies a classic newsvendor problem with no BCR and PTR terms.

Proof. For the exact proof procedure, please refer to Lemma 1 in the work of [24].

Lemma 2. In Model 0 , higher values of $p, s$, and $v$ increase $\hat{x}_{0}(\alpha)$, while higher value of $c$ decrease $\hat{x}_{0}(\alpha)$ for the newsvendor parameters. For CAT parameters, higher values of $b$ and $\alpha$ decrease $\hat{x}_{0}(\alpha)$, while the parameters $a$ and $K$ do not affect $\hat{x}_{0}(\alpha)$. 
Proof. The Lemma 2 is about the sensitivity analysis for the impacts of the newsvendor and CAT parameters in Model 0 . For this sensitivity analysis, our proof idea is to directly use supermodularity (refer to the work of [38]) and implicit function theorem at the results of Lemma 1.

\subsection{Analytical Results for Models 1 and 2}

Now let us resume our analysis with Model 1 where its objective function, $\mathbb{E}\left[\Pi_{1}(x, D)\right]$, is the same as $\mathbb{E}[\Pi(x, D)]$ in Equation (2) as we simply re-denote $\Pi_{1}(x, D) \equiv \Pi(x, D)$. Then, Model 1 can be re-described as follows:

$$
\text { Model 1: } \max _{x \geq 0} \mathbb{E}\left[\Pi_{1}(x, D)\right]=\mathbb{E}\left[p * \min \{D, x\}-c x+v(x-D)^{+}-s(D-x)^{+}-\alpha(a+b x-K)^{+}\right] .
$$

Then, the following Theorem 1 shows the closed-form optimal solution in Model 1.

Theorem 1. If $K \geq a+b \hat{x}_{0}(0)$, the optimal solution of Model $1 \hat{x}_{1}(\alpha)=\hat{x}_{0}(0)=F_{D}^{-1}\left(\frac{p+s-c}{p+s-v}\right)$. Otherwise, if $K<a+b \hat{x}_{0}(0), \hat{x}_{1}(\alpha)=\hat{x}_{0}(\alpha)=F_{D}^{-1}\left(\frac{p+s-c-\alpha b}{p+s-v}\right)$.

Proof. First of all, we can take the first derivative of Equation (5) as follows.

$$
\frac{d \mathbb{E}\left[\Pi_{1}(x, D)\right]}{d x}=\left\{\begin{array}{r}
(p+s-c)-(p+s-v) F_{D}(x), x \leq(K-a) / b \\
(p+s-c-\alpha b)-(p+s-v) F_{D}(x), x>(K-a) / b
\end{array} .\right.
$$

Then, a necessary condition of the optimal solution is the point of $x$ where the value of the first-order condition is equal to zero (i.e., $\left.\frac{d \mathbb{E}\left[\Pi_{1}(x, D)\right]}{d x}=0\right)$. Now, the first derivative may take two different forms depending on the value of $x$ with the given $C A T$ parameters. Then, we can derive the optimal solution for each case, respectively, by equating the first derivative to zero. For a sufficient condition of the optimal solution in an unconstrained maximization problem, the value of the second-order condition is always non-positive. Then,

$$
\frac{d^{2} \mathbb{E}\left[\Pi_{1}(x, D)\right]}{d x^{2}}=-(p+s-v) f_{D}(x) \leq 0,
$$

because $p+s-v>0$ and $f_{D}(x) \geq 0$ for every $x \geq 0$. Although our objective function is actually piecewise linear and quasi-concave, the existence condition of our optimal solution does not change.

Next, we modify our Model 1 slightly to extend it to another model, Model 2, as follows. In Model 1, we assume that the amounts of the remaining emission right $(K-a-b x)^{+}$would be discarded. Then, in Model 2, we can obtain some value $\beta$ from the remaining emission right per unit, but $\alpha \geq \beta$. For this reason, note that this Model 2 is a more generalized problem of Model 1 previously studied. That is, if $\beta=0$, Model 2 is equivalent to Model 1 . On the other hand, if $\alpha=\beta$, Model 2 is equivalent to Model 1, but the BCR term is replaced by the PTR term. As these two special cases do not add a big theoretical contribution, we focus on Model 2 with the condition $\alpha>\beta$. Thus, we redefine a new profit function $\Pi_{2}(x, D)$ for Model 2 as follows:

$$
\Pi_{2}(x, D)=p * \min \{D, x\}-c x+v(x-D)^{+}-s(D-x)^{+}-\alpha(a+b x-K)^{+}+\beta(K-a-b x)^{+} .
$$

Similarly, we set up Model 2, which is $\max _{x \geq 0} \mathbb{E}\left[\Pi_{2}(x, D)\right]$, as follows:

Model 2: $\max _{x \geq 0} \mathbb{E}\left[p * \min \{D, x\}-c x+v(x-D)^{+}-s(D-x)^{+}-\alpha(a+b x-K)^{+}+\beta(K-a-b x)^{+}\right]$.

Then, we will show the closed-form optimal solution in Model 2 from the following Theorem 2. 
Theorem 2. If $K \geq a+b \hat{x}_{0}(\beta)$, the optimal solution of Model 2 is $\hat{x}_{2}(\alpha, \beta)=\hat{x}_{0}(\beta)=F_{D}^{-1}\left(\frac{p+s-c-\beta b}{p+s-v}\right)$. Otherwise, if $K<a+b \hat{x}_{0}(\beta), \hat{x}_{2}(\alpha, \beta)=\hat{x}_{0}(\alpha)=F_{D}^{-1}\left(\frac{p+s-c-\alpha b}{p+s-v}\right)$.

Proof. First of all, we can take the first derivative of Equation (9) as follows.

$$
\frac{d \mathbb{E}[\Pi(x, D)]}{d x}=\left\{\begin{array}{l}
(p+s-c-\beta b)-(p+s-v) F_{D}(x), x \leq(K-a) / b \\
(p+s-c-\alpha b)-(p+s-v) F_{D}(x), x>(K-a) / b
\end{array} .\right.
$$

Then, we can obtain the second derivative of Equation (9), similarly obtained at Equation (6), as follows:

$$
\frac{d^{2} \mathbb{E}[\Pi(x, D)]}{d x^{2}}=-(p+s-v) f_{D}(x) \leq 0 .
$$

Then, the remaining proof procedure is similar to that of Theorem 1.

\subsection{Discussions}

In this subsection, we analyze the closed-form solutions in Theorems 1 and 2 to summarize the managerial interpretations and implications as below. We conduct a sensitivity analysis through a comparative static analysis for the impacts of newsvendor and CAT parameters on the optimal solutions in Models 1 and 2. According to the definition of comparative static analysis, we choose a (newsvendor or CAT) parameter (or exogenous variable) from each model, and then see how changes in the value of the selected parameter affect the optimal solution, which is an endogenous variable, while all other parameters still keep the same values [39]. In particular, by conducting the analysis, we aim to verify whether the direction of the impact is monotone (increasing or decreasing) or indeterminate.

In addition, our goals through comparative static analysis are primarily focused on the analysis of the general effects of the parameters in our research models on optimal production quantity. In other words, the parameters of the research models do not directly affect the optimal objective value, the expected profit. Rather, we can see that there exists a mediating variable, optimal production quantity, which is also a decision variable. That is, the final relationship between the parameters and expected profit is determined by a combination of the two relationships in which the parameters of the study model first affect the optimal solution and then the optimal solution again affects the expected profit. Then, owing to the definition of comparative static analysis in the work of [39], our analytical method is to analyze only the first relationship, which has been a common analytical method in POM literature. In addition, another reason to consider the first relationship only is that the expected profit is a concave function of an optimal solution. It implies that the second relationship does not preserve the (monotone) direction of the first relationship. As a result, because of the mixed effects of the first and second relationships, the direction of the impact in the final relationship would become overly complicated without additional meaningful implications nor insights to our decision, production quantity. Thus, we provide our discussions based on the analysis of the first relationship as below.

Observation 1. In Model 1, let us first consider the impacts of the newsvendor parameters. Then, the direction of the impact of $p$ is not monotone. In fact, we split totally three cases: (1) $K>a+b \hat{x}_{0}(0)$; (2) $K=a+b \hat{x}_{0}(0)$; and (3) $K<a+b \hat{x}_{0}(0)$. For the sensitivity analysis of $p$, we start with a sufficiently small value of $p$ where it satisfies case 1 . Then, in case $1, p$ increases the optimal solution, $\hat{x}_{0}(0)$. When we increase the value of $p$ continuously, it starts to satisfy case 2 , instead of case 1 where $K=a+b \hat{x}_{0}(0)$. Here, the value of $\hat{x}_{1}(0)$ falls vertically in case 2 . When we again continuously increase the value of $p$, it finally satisfies case 3 , and increased $p$ leads to higher optimal solution $\hat{x}_{0}(\alpha)$ afterwards. Next, the parameters $v$ and $s$ have the same directions as the impact of $p$ on $\hat{x}_{1}(0)$ and $\hat{x}_{1}(\alpha)$ for all three cases. For the impact of $c$, the direction is reversed. That is, when we start with a sufficiently small value of $c$ where it satisfies case 3 . Then, in case $1, p$ decreases the optimal solution, 
$\hat{x}_{0}(\alpha)$. When we increase the value of $c$ continuously, it starts to satisfy case 2 . Here, the value of $\hat{x}_{1}(\alpha)$ rises vertically in case 2 . When we continuously increase the value of $c$, it finally satisfies case 1 , and increased $c$ leads to lower optimal solution $\hat{x}_{0}(\alpha)$ afterwards.

Observation 2. Next, we consider the impacts of the CAT parameters in Model 1. For the sensitivity analysis of $a$, it is found that when the value of $a$ is sufficiently small, it initially satisfies case 1 . Then, in case $1, a$ has no effects on the optimal solution, $\hat{x}_{0}(0)$. When we increase the value of $a$ continuosly, it starts to satisfy case 2 . Here, the value of $\hat{x}_{1}(0)$ falls vertically in case 2 . When we again continuously increase the value of $a$, it finally satisfies case 3 , and increased $a$ also has no effects on the optimal solution $\hat{x}_{0}(\alpha)$ afterwards. For the sensitivity analysis of $K$, the direction of the impact is reversed compared with that of $a$. That is, it initially satisfies case 3 where $a$ has no effects on the optimal solution, $\hat{x}_{0}(\alpha)$. Afterwards, it satisfies case 2 when the value of $\hat{x}_{1}(\alpha)$ rises vertically. Finally, it finally satisfies case 1 , and increased $K$ also has no effects on the optimal solution $\hat{x}_{0}(0)$. For the sensitivity analysis of $b$, it initially satisfies case 1 , and $a$ has no effects on the optimal solution, $\hat{x}_{0}(0)$. When we increase the value of $b$ continuously, it starts to satisfy case 2 , and the value of $\hat{x}_{1}(0)$ falls vertically in case 2 . When we again continuously increase the value of $b$, it finally satisfies case 3 , and increased $b$ leads to lower optimal solution, $\hat{x}_{0}(\alpha)$. In the last case, for the sensitivity analysis of $\alpha$, the direction of the impact depends on whether it satisfies cases 1 and 2 or 3 . If it satisfies cases 1 and 2, increased $\alpha$ has no effects on the optimal solution, $\hat{x}_{0}(\alpha)$. Otherwise, if it satisfies case 3 , increased $\alpha$ leads to lower optimal solution, $\hat{x}_{0}(\alpha)$. Note that whether it satisfies cases 1 and 2 or 3 is determined not by the value of $\alpha$, but by the values of all other newsvendor and BCR parameters.

Observation 3. From the results of Theorem 1, the optimal solution in Model 1 has a jump at the point of satisfying the condition $K=a+b \hat{x}_{0}(0)$. Then, the (absolute) size of the jump is $F_{D}^{-1}\left(\frac{p+s-c}{p+s-v}\right)-F_{D}^{-1}\left(\frac{p+s-c-\alpha b}{p+s-v}\right)$. This implies that a higher value of $\alpha$ tends to make the jump size bigger. Note that it does not mean increasing the jump size proportionally, in general. In fact, only when demand is represented with uniform distribution does it lead to a proportional change of the jump size in the value of $\alpha$. Also note that when $\alpha=0$ trivially, the jump size shrinks to zero. In other words, there is no kinked point of the optimal solution in the change of model parameters. In fact, when $\alpha=0$, the $B C R$ terms disappears, that is, the objective function only has newsvendor parameters, which is the case of the well-known classic newsvendor problem in literature.

Observation 4. In Model 2, we also have three cases, similarly done at Model 1. However, in Model 2, the boundary point between cases 1 and 3 satisfies the condition $K=a+b \hat{x}_{0}(\beta)=$ $a+b F_{D}^{-1}\left(\frac{p+s-c-\beta b}{p+s-v}\right)$ at case 2 , which is different from the boundary point $K=a+b \hat{x}_{0}(0)=$ $a+b F_{D}^{-1}\left(\frac{p+s-c}{p+s-v}\right)$ in Model 1. Then, for the impacts of both the newsvendor $(p, c, v, s)$ and $C A T$ $(K, a, b, \alpha)$ parameters on the optimal solution, $\hat{x}_{2}(\alpha, \beta)$, the results of the sensitivity analysis are the same as those in Model 1 only, except for $b$ and $\beta$. For the impact of $b$ in Model 2, it initially satisfies case 1 , and $b$ decreases $\hat{x}_{2}(\alpha, \beta)$. When we increase the value of $b$ continuously, it starts to satisfy case 2 , and the value of $\hat{x}_{2}(\alpha, \beta)$ falls vertically. When we again continuously increase the value of $b$, it finally satisfies case 3 , and $b$ decreases $\hat{x}_{2}(\alpha, \beta)$ relatively more steeply compared with the change in case 1 . For the impact of $\beta$, it initially satisfies case 3 , and $\beta$ has no effects on $\hat{x}_{2}(\alpha, \beta)$. When we increase the value of $\beta$ continuously, it starts to satisfy case 2 , the value of $\hat{x}_{2}(\alpha, \beta)$ falls vertically. When we again continuously increase the value of $\beta$, it finally satisfies case 1 , and increased $\beta$ leads to lower $\hat{x}_{2}(\alpha, \beta)$.

Observation 5. From the results of Theorem 2, the optimal solution in Model 2 has a jump at the point of satisfying the condition $K=a+b \hat{x}_{0}(\beta)$. Then, the (absolute) size of the jump is $F_{D}^{-1}\left(\frac{p+s-c-\beta b}{p+s-v}\right)-$ $F_{D}^{-1}\left(\frac{p+s-c-\alpha b}{p+s-v}\right)$. Here, the condition $\alpha>\beta$ implies that the trading values of emission rights are higher than those of emission rights when to purchase emission rights compared with the case to sell the emission rights. Similar to Observation 3 in the analysis of Model 1, a higher difference of $\alpha-\beta$ tends to make the jump size bigger. 
Observation 6. Suppose that demand uncertainty is removed in our models. That is, the variability of the demand shrinks to be zero, while the expected value of demand remains the same. Finally, we obtain the corresponding deterministic models with fixed demand. In this case, the optimal solutions for Models 0,1 , and 2 are equal to $D$ (fixed demand), where the mismatch costs are minimized between supply and demand. This implies that the original stochastic models and their corresponding deterministic models are structurally very different.

Observation 7. For analytical tractability, we focus on two single-stage, single-period, and single-echelon models with only one product in our work. Because of these simple characteristics in the models studied, we are able to obtain the closed-form optimal solutions as above, respectively. For the generalizability of our work to the corresponding multi-product model, let us think of a two-product case with $\Pi_{1}$ (the profit function partially obtained from product 1 ), $\Pi_{2}$ (the profit function partially obtained from product 2 ), and $\Pi_{0}$ (the total profit function from products 1 and 2). As an expected-value operator satisfies additivity, $\mathbb{E}\left[\Pi_{0}\right] \equiv \mathbb{E}\left[\Pi_{1}\right]+\mathbb{E}\left[\Pi_{2}\right]$, which implies that the expected value of totality is equivalent to the total sum of the expected value of the part. As this relationship can easily be shown to be valid for an arbitrary number of products, the multi-product model is always decomposable as long as our optimization models do not have additional constraints. That is, our analysis leads to the same optimal solution for each product whether we consider a single multi-product model as a whole or not multiple single-product models for each product. Thus, our results in a single-product model are generalizable for the case with multiple products under sound conditions.

However, once the model becomes more complicated in other ways (e.g., multi-stage, multi-period, and multi-echelon models), the extended models are not separable, in general. Thus, our's and extended models are clearly different from each other, inevitably leading to different optimal solutions. In fact, it is believed that every single model in this research stream embodies some certain problem in real world cases, so even a very simple model seems to be worth studying because of its own unique features and characteristics that exist in reality.

In summary, we provide Table 1 to compare the sensitivity analysis results in the above five Observations 1 through 5 as follows. In Table $1, \uparrow$ (or $\downarrow$ ) means monotone increasing (or decreasing), while $\downarrow \downarrow$ implies relatively decreasing more steeply than $\downarrow$. In addition, FV (or RV) means falling (or rising) vertically while NE and NA imply no effects and not available, respectively. The results of Table 1 show that the analytical results in Models 0,1 , and 2 are closely related but different from each other. In order to highlight the differences, we compare the sensitivity analysis results in Model 1 with those in Model 0, and then see the differences between Model 1 and 2. First, in the comparison between the results of Models 0 and 1, the most distinguishing characteristic is that the optimal solution in Model 1 is jumping up or down at case 2 with $K=a+b \hat{x}_{0}(0)$ for all the newsvendor parameters $(p, v, s, c)$ and some of the BCR parameters $(K, a)$. Then, the directions of the impacts of $b$ and $\alpha$ are the same as in case 3 , but different in to those in cases 1 and 2. Second, in the comparison between the results of Models 1 and 2, the location of the kinked point in case 2 changes to another point satisfying the condition, $K=a+b \hat{x}_{0}(\beta)$, from the point satisfying the condition, $K=a+b \hat{x}_{0}(0)$. Next, all the directions of the impacts of all the newsvendor parameters $(p, v, s, c)$ and some of the BCR parameters $(K, a, \alpha)$ are the same as each other, while the direction of the impact of $b$ is the same as in case 2, but different to that in cases 1 and 3. In the last case, $\beta$ is a new BCR parameter in Model 2, so the direction of the impact of $\beta$ is freshly studied in Model 2 . 
Table 1. Results of sensitivity analysis in Models 0, 1, and 2. FV—falling vertically; RV—rising vertically; NE—no effect; NA—not available; $\mathrm{BCR}$ - baseline credit regulation.

\begin{tabular}{|c|c|c|c|c|}
\hline Parameter & & Model 0 & Model 1 & Model 2 \\
\hline \multirow{4}{*}{ Newsvendor } & $p$ & $\uparrow$ & $\begin{array}{c}\text { If } K>a+b \hat{x}_{0}(0), \uparrow \\
\text { At } K=a+b \hat{x}_{0}(0), \mathrm{FV} \\
\text { If } K<a+b \hat{x}_{0}(0), \uparrow\end{array}$ & $\begin{array}{c}\text { If } K>a+b \hat{x}_{0}(\beta), \uparrow \\
\text { At } K=a+b \hat{x}_{0}(\beta), \mathrm{FV} \\
\text { If } K<a+b \hat{x}_{0}(\beta), \uparrow\end{array}$ \\
\hline & $v$ & $\uparrow$ & $\begin{array}{l}\text { If } K>a+b \hat{x}_{0}(0), \uparrow \\
\text { At } K=a+b \hat{x}_{0}(0), \mathrm{FV} \\
\text { If } K<a+b \hat{x}_{0}(0), \uparrow\end{array}$ & $\begin{array}{c}\text { If } K>a+b \hat{x}_{0}(\beta), \uparrow \\
\text { At } K=a+b \hat{x}_{0}(\beta), \mathrm{FV} \\
\text { If } K<a+b \hat{x}_{0}(\beta), \uparrow\end{array}$ \\
\hline & $s$ & $\uparrow$ & $\begin{array}{l}\text { If } K>a+b \hat{x}_{0}(0), \uparrow \\
\text { At } K=a+b \hat{x}_{0}(0), \mathrm{FV} \\
\text { If } K<a+b \hat{x}_{0}(0), \uparrow\end{array}$ & $\begin{array}{c}\text { If } K>a+b \hat{x}_{0}(\beta), \uparrow \\
\text { At } K=a+b \hat{x}_{0}(\beta), \mathrm{FV} \\
\text { If } K<a+b \hat{x}_{0}(\beta), \uparrow\end{array}$ \\
\hline & $c$ & $\downarrow$ & $\begin{array}{c}\text { If } K<a+b \hat{x}_{0}(0), \downarrow \\
\text { At } K=a+b \hat{x}_{0}(0), \mathrm{RV} \\
\text { If } K>a+b \hat{x}_{0}(0), \downarrow\end{array}$ & $\begin{array}{l}\text { If } K<a+b \hat{x}_{0}(\beta), \downarrow \\
\text { At } K=a+b \hat{x}_{0}(\beta), \mathrm{RV} \\
\text { If } K>a+b \hat{x}_{0}(\beta), \downarrow\end{array}$ \\
\hline \multirow{5}{*}{$B C R$} & K & $\mathrm{NE}$ & $\begin{array}{l}\text { If } K<a+b \hat{x}_{0}(0), \mathrm{NE} \\
\text { At } K=a+b \hat{x}_{0}(0), \mathrm{RV} \\
\text { If } K>a+b \hat{x}_{0}(0), \mathrm{NE}\end{array}$ & $\begin{array}{l}\text { If } K<a+b \hat{x}_{0}(\beta), \mathrm{NE} \\
\text { At } K=a+b \hat{x}_{0}(\beta), \mathrm{RV} \\
\text { If } K>a+b \hat{x}_{0}(\beta), \mathrm{NE}\end{array}$ \\
\hline & $a$ & NE & $\begin{array}{l}\text { If } K>a+b \hat{x}_{0}(0), \mathrm{NE} \\
\text { At } K=a+b \hat{x}_{0}(0), \mathrm{FV} \\
\text { If } K<a+b \hat{x}_{0}(0), \mathrm{NE}\end{array}$ & $\begin{array}{l}\text { If } K>a+b \hat{x}_{0}(\beta), \mathrm{NE} \\
\text { At } K=a+b \hat{x}_{0}(\beta), \mathrm{FV} \\
\text { If } K<a+b \hat{x}_{0}(\beta), \mathrm{NE}\end{array}$ \\
\hline & $b$ & $\downarrow$ & $\begin{array}{c}\text { If } K>a+b \hat{x}_{0}(0), \mathrm{NE} \\
\text { At } K=a+b \hat{x}_{0}(0), \mathrm{FV} \\
\text { If } K<a+b \hat{x}_{0}(0), \downarrow\end{array}$ & $\begin{array}{l}\text { If } K>a+b \hat{x}_{0}(\beta), \downarrow \\
\text { At } K=a+b \hat{x}_{0}(\beta), \mathrm{FV} \\
\text { If } K<a+b \hat{x}_{0}(\beta), \downarrow \downarrow\end{array}$ \\
\hline & $\alpha$ & $\downarrow$ & $\begin{array}{l}\text { If } K \geq a+b \hat{x}_{0}(0), \mathrm{NE} \\
\text { If } K<a+b \hat{x}_{0}(0), \downarrow\end{array}$ & $\begin{array}{l}\text { If } K \geq a+b \hat{x}_{0}(\beta), \mathrm{NE} \\
\text { If } K<a+b \hat{x}_{0}(\beta), \downarrow\end{array}$ \\
\hline & $\beta$ & NA & NA & $\begin{array}{c}\text { If } K<a+b \hat{x}_{0}(\beta), \mathrm{NE} \\
\text { If } K=a+b \hat{x}_{0}(\beta), \mathrm{FV} \\
\text { If } K>a+b \hat{x}_{0}(\beta), \downarrow\end{array}$ \\
\hline
\end{tabular}

\section{Conclusions}

As both sustainability and open innovation have become pivotal subjects in the fourth industrial revolution, or industry 4.0 [40-43], emission abatement in manufacturing firms has also been a hot topic in the literature. In particular, this topic corresponds to the sustainable production through an open innovation approach. Thus, it is necessary to actively consider various factors involved in eco-friendly production in determining the production activities and process in manufacturing companies. As described in the works of [4,44], a conceptual framework for this new trend is the triple bottom line (TBL) approach, by which manufacturing companies can capture the social and economic goals simultaneously. In addition, the CAT system helps manufacturers to determine their production decision under a collaboration with external vendors, so it is in harmony with an open innovation approach. Note that in the era of the fourth industrial revolution, companies face a greater risk in their business environments. Then, open innovation can be a good approach to improve the decision making of newsvendors under risk by taking a balance from internal and external resources in companies. That is, we can reduce our vulnerability with risk pooling in supply chain participants $[45,46]$.

In this paper, we consider an emission decision problem with BCR. Then, in this stream of literature, PTR and BCR have been proposed as the actual, implementable policies in practice. Both of these approaches are in good harmony with innovative government-led policies that fit well with open innovation as well as the fourth industrial revolution, in that manufacturing companies pursue sustainable production with reasonable economic motivation. Nevertheless, the previous studies in the literature focus on PTR but not on BCR. In order to fill this gap of knowledge, this study aims to support the smart decision making of innovative manufacturing firms by presenting the optimization 
model and its analytical result that can maximize the operational efficiency of the firms under the BCR basis.

Now, we can summarize our theoretical contributions as follows. We provide our methodology of mathematical modeling to analyze and compare BCR and PTR approaches. Then, with a mathematical modeling and analysis, the objective function is to maximize an expected profit because our problem is a stochastic optimization problem with demand uncertainty. Then, our problem is formulated as a piecewise linear and quasi-concave function due to a kinked point between the total emission amount and emission cap. Then, we analyze the two models, which are different from the trading values in the remaining emission right per unit. As a result, we show that the optimal solution exists uniquely. Afterwards, we conduct a sensitivity analysis to perform a comparative static analysis. By doing so, we can examine how the optimal solution can be influenced by the model (newsvendor and $C A T)$ parameters.

Meanwhile, a practical contribution of our study is that our mathematical models have immediate applicability for BCR and PTR approaches, which are two famous CAT policies that exist in reality. Thus, our study can add a valuable feature in POM literature with a direct application to sustainable production in manufacturing companies. From that point, our work can be classified more closely to an application modeling rather than theoretical modeling. Although the analytical results are quite similar, these two CAT optimization models with BCR and PTR approaches are not the same and can be applied for different pollutants. Note that the previous studies for the CAT systems have focused on the PTR approach, which is mainly useful to analyze the emission problems for carbon dioxide. Although this phenomenon is consistent with the trend in this stream of research, there still exists a knowledge gap in literature for the analysis of emissions decision problems in other non-carbon major pollutants such as sulfur dioxides, mercury, and nitrogen compounds. From that sense, our study has another practical contribution in the literature to resolve a well-known pollution hotspot problem in this research stream [47].

For future directions of our work, we may consider the following topics. First, we consider a newsvendor approach to hypothesize a stochastic demand of end customers in the models studied. Then, it can be extended to an SCM problem with more than a single-echelon problem. Thus, we can add SCM issues including coordination contracts in decentralized and centralized SCM problems. Second, similar to the first future direction as above, we can consider some extended models with multi-stage or multi-period models in this research stream. It is obvious that such extended models are totally different from our models to be applicable for certain production processes in manufacturing companies. Unfortunately, it seems that we are unable to derive such strong analytical results for these extended models compared with the results in this work. Then, rather than sensitivity analysis through a comparative static analysis, a new analytical methodology may be needed for the models, such as simulation-based numerical experiments or heuristic algorithms. Third, some additional characteristics in pollution reduction can be included in the model such as greening investment. In these cases, the greening investment may cause the relevant demand expansion in the model. In the last, but not the least case, the risk preferences for inventory managers may take other risk attitudes than risk neutrality. Some possible risk preferences could be risk aversion, loss aversion, and so on [48].

Author Contributions: All authors significantly contributed to the scientific study and writing. S.C. contributed to the overall idea, model formulation and analysis, and writing of the manuscript; K.P. contributed to the detailed writing and discussions on open innovation and sustainability of operation system and manufacturing firms, as well as preparation of publishing the paper; S.-O.S. contributed to the detailed writing and concepts on the model.

Funding: This research received no external funding.

Conflicts of Interest: The authors declare no conflict of interest. 


\section{References}

1. Lee, S.D. Wintering habitat use pattern of red-crowned cranes in the Korean demilitarized zone. J. Open Innov. Technol. Mark. Complex. 2018, 4, 58. [CrossRef]

2. Dong, C.; Shen, B.; Chow, P.S.; Yang, L.; Ng, C.T. Sustainability investment under cap-and-trade regulation. Ann. Oper. Res. 2016, 240, 509-531. [CrossRef]

3. Alsaffar, A.J.; Raoufi, K.; Kim, K.Y.; Okudan Kremer, G.E.; Haapala, K.R. Simultaneous consideration of unit manufacturing processes and supply chain activities for reduction of product environmental and social impacts. ASME J. Manuf. Sci. Eng. 2016, 138, 101009. [CrossRef]

4. Kleindorfer, P.R.; Singhal, K.; Wassenhove, L.N.V. Sustainable operations management. Prod. Oper. Manag. 2005, 14, 482-492. [CrossRef]

5. Zhang, J.J.; Nie, T.F.; Du, S.F. Optimal emission-dependent production policy with stochastic demand. Int. J. Soc. Syst. Sci. 2011, 3, 21-39. [CrossRef]

6. Boswall, J.; Lee, R. Economics, Ethics and the Environment; Routledge-Cavendish: London, UK, 2002; pp. $62-66$. ISBN 9781135597924.

7. Du, S.; Ma, F.; Fu, Z.; Zhu, L.; Zhang, J. Game-theoretic analysis for an emission-dependent supply chain in a 'cap-and-trade' system. Ann. Oper. Res. 2015, 228, 135-149. [CrossRef]

8. Hua, G.; Cheng, T.C.E.; Wang, S. Managing carbon footprints in inventory control. Int. J. Prod. Econ. 2011, 132, 178-185. [CrossRef]

9. Burton, E.S.; Sanjour, W. A simulation approach to air pollution abatement program planning. Socioecon. Plann. Sci. 1970, 4, 147-150. [CrossRef]

10. Rosen, H.S.; Gayer, T. Public Finance, 10th ed.; McGraw-Hill Education: New York, NY, USA, 2013 ; p. 90. ISBN 9780078021688.

11. Lu, Y.; Zhu, X.; Cui, Q. Effectiveness and equity implications of carbon policies in the United States construction industry. Build. Environ. 2012, 49, 259-269. [CrossRef]

12. Chomitz, K.M. Evaluating Carbon Offsets from Forestry and Energy Projects: How Do They Compare? World Bank Policy Research Working Paper No. 2357; World Bank: Washington, DC, USA, 1999.

13. Chesbrough, H.W. Open Innovation: The New Imperative for Creating and Profiting from Technology; Harvard Business School Press: Boston, MA, USA, 2003; ISBN 9781578518371.

14. Burney, N.E. Carbon Tax and Cap-and-Trade Tools: Market-Based Approaches for Controlling Greenhouse Gases; Nova Science Publishers: New York, NY, USA, 2010; ISBN 9781608761.

15. Ramseur, J.L. Estimating Offset Supply in a Cap-and-Trade Program; Congressional Research Service: Washington, DC, USA, 2011; retrieved 15 February 2011.

16. Map: Pollution Hotpots. Available online: https://news.bbc.co.uk/2/hi/science/nature/4083331.stm (accessed on 10 December 2018).

17. Benjaafar, S.; Li, Y.Z.; Daskin, M. Carbon footprint and the management of supply chains: Insights from simple models. IEEE Trans. Autom. Sci. Eng. 2013, 10, 99-116. [CrossRef]

18. Ye, B.; Jiang, J.J.; Miao, L.; Li, J.; Peng, Y. Innovative carbon allowance allocation policy for the shenzhen emission trading scheme in China. Sustainability 2016, 8, 3. [CrossRef]

19. Weitzman, M.L. Prices vs. quantities. Rev. Econ. Stud. 1974, 41, 477-491. [CrossRef]

20. Cropper, M.L.; Oates, W.E. Environmental economics: A survey. J. Econ. Lit. 1992, 30, 675-740.

21. Hepburn, C. Regulation by prices, quantities or both: A review of instrument choice. Oxf. Rev. Econ. Policy 2006, 22, 226-247. [CrossRef]

22. Swami, S.; Shah, J. Channel coordination in green supply chain management. J. Oper. Res. Soc. 2013, 64, 336-351. [CrossRef]

23. Qin, J.; Bai, X.; Xia, L. Sustainable trade credit and replenishment policies under the cap-and-trade and carbon tax regulations. Sustainability 2015, 7, 16340-16361. [CrossRef]

24. Chen, X.; Gong, W.; Wang, F. Managing carbon footprints under the trade credit. Sustainability 2017, 9, 1235. [CrossRef]

25. Fang, X.; Du, Y.; Qiu, Y. Reducing carbon emissions in a closed-loop production routing problem with simultaneous pickups and deliveries under carbon cap-and-trade. Sustainability 2017, 9, 2198. [CrossRef]

26. Wang, X.; Xue, M.; Xing, L. Analysis of carbon emission reduction in a dual-channel supply chain with cap-and-trade regulation and low-carbon preference. Sustainability 2018, 10, 580. [CrossRef] 
27. Battini, D.; Calzavara, M.; Isolan, I.; Sgarbossa, F.; Zangaro, F. Sustainability in material purchasing: A multi-objective economic order quantity model under carbon trading. Sustainability 2018, 10, 4438. [CrossRef]

28. Choi, S. A loss-averse newsvendor with cap-and-trade carbon emissions regulation. Sustainability 2018, 10, 2126. [CrossRef]

29. Yuan, B.; Gu, B.; Guo, J.; Xia, L.; Xu, C. The optimal decisions for a sustainable supply chain with carbon information asymmetry under cap-and-trade. Sustainability 2018, 10, 1002. [CrossRef]

30. Arrow, K.; Harris, T.; Marshak, J. Optimal inventory policy. Econometrica 1951, 19, 250-272. [CrossRef]

31. Khouja, M. The single-period (news-vendor) problem: Literature review and suggestions for future research. Omega 1999, 27, 537-553. [CrossRef]

32. Qin, Y.; Wang, R.; Vakharia, A.J.; Chen, Y.; Seref, M.M.H. The newsvendor problem: Review and directions for future research. Eur. J. Oper. Res. 2011, 213, 361-374. [CrossRef]

33. Smith, S.J.; Pitcher, H.; Wigley, T.M.L. Global and regional anthropogenic sulfur dioxide emissions. Glob. Planet Chang. 2001, 29, 99-109. [CrossRef]

34. Smith, S.J.; van Aardenne, J.; Klimont, Z.; Andres, R.J.; Volke, A.; Arias, S.D. Anthropogenic sulfur dioxide emissions: 1850-2005. Atmos. Chem. Phys. 2011, 11, 1101-1116. [CrossRef]

35. Loredo, J.; Ordóñez, A.; Álvarez, R. Environmental impact of toxic metals and metalloids from the Muñón Cimero mercury-mining area (Asturias, Spain). J. Hazard. Mater. 2006, A136, 455-467. [CrossRef]

36. Aneja, V.P.; Roelle, P.A.; Murray, G.C.; Southerland, J.; Erisman, J.W.; Fowler, D.; Asman, W.A.H.; Patni, N. Atmospheric nitrogen compounds II: Emissions, transport, transformation, deposition and assessment. Atmos. Environ. 2001, 35, 1903-1911. [CrossRef]

37. Snyder, L.V.; Shen, Z.M. Fundamentals of Supply Chain Theory; Wiley: Hoboken, NJ, USA, 2011; ISBN 9780470521304.

38. Topkis, D. Supermodularity and Complementarity; Princeton University Press: Princeton, NJ, USA, 1998; ISBN 9780691032443.

39. Chiang, A.C.; Wainwright, K. Fundamental Methods of Mathematical Economics, 4th ed.; McGraw-Hill Education: New York, NY, USA, 2004; ISBN 9780070109100.

40. Yun, J.J. How do we conquer the growth limits of capitalism? Schumpeterian dynamics of open innovation. J. Open Innov. Technol. Mark. Complex. 2015, 1, 17. [CrossRef]

41. Yun, J.J.; Won, D.K.; Park, K.B. Dynamics from open innovation to evolutionary change. J. Open Innov. Technol. Mark. Complex. 2016, 2, 7. [CrossRef]

42. Lee, S.D.; Kwon, S.S. Carbon sequestration in the urban areas of Seoul with climate change: Implication for open innovation in environmental industry. J. Open Innov. Technol. Mark. Complex. 2018, 4, 48. [CrossRef]

43. Shim, S.O.; Park, K.B.; Choi, S. Innovative production scheduling with customer satisfaction based measurement for the sustainability of manufacturing firms. Sustainability 2017, 9, 2249. [CrossRef]

44. Lee, J.; Lee, M.L.; Park, M. A newsboy model with quick response under sustainable carbon cap-n-trade. Sustainability 2018, 10, 1410. [CrossRef]

45. Choi, S.; Park, K.B.; Shim, S.O. Comparing validity of risk measures on newsvendor models in open innovation perspective. J. Open Innov. Technol. Mark. Complex. 2018, 4, 1. [CrossRef]

46. Yoon, S.; Jeong, S. Effects to implement the open-innovation coordinative strategies between manufacturer and retailer in reverse supply chain. J. Open Innov. Technol. Mark. Complex. 2017, 3, 2. [CrossRef]

47. Morag-Levine, N. The problem of pollution hotspots: Pollution markets, coase, and common law. Cornell J. Law Public Policy 2007, 17, 161-199.

48. Schweitzer, M.E.; Cachon, G. Decision bias in the newsvendor problem with a known demand distribution: Experimental evidence. Manag. Sci. 2000, 46, 404-420. [CrossRef]

(C) 2019 by the authors. Licensee MDPI, Basel, Switzerland. This article is an open access article distributed under the terms and conditions of the Creative Commons Attribution (CC BY) license (http://creativecommons.org/licenses/by/4.0/). 